\title{
New Largest Known Graphs of Diameter 6 *
}

\author{
Guillermo Pineda-Villavicencio ${ }^{1,2, \dagger}$ José Gómez ${ }^{3, \ddagger}$ \\ Mirka Miller ${ }^{4,5, \S}$ Hebert Pérez-Rosés ${ }^{4, \boldsymbol{q}}$ \\ ${ }^{1}$ School of Information Technology and Mathematical Sciences \\ University of Ballarat, Australia \\ ${ }^{2}$ Department of Computer Science \\ University of Oriente, Cuba \\ ${ }^{3}$ Department of Applied Mathematics IV \\ Technical University of Catalonia, Spain \\ ${ }^{4}$ School of Electrical Engineering and Computer Science \\ The University of Newcastle, Australia \\ ${ }^{5}$ Department of Mathematics \\ University of West Bohemia, Czech Republic
}

\begin{abstract}
In the pursuit of obtaining largest graphs of given maximum degree $\Delta$ and diameter $D$, many construction techniques have been developed. Compounding of graphs is one such technique. In this paper, by means of the compounding of complete graphs into a Moore bipartite graph of diameter 6 , we obtain a family of large graphs of the same diameter. For
\end{abstract}

${ }^{*}$ Research supported in part by the Australian Research Council grant ARC DP0450294, by the Spanish Ministry of Education under the project MTM2005-08990-C02-01 and by the Catalan Research Council under the project 2005 SGR00256.

\begin{tabular}{l|l|} 
g.pinedav@gmail.com \\
\hline jgomez@ma4.upc.edu \\
mirka.miller@newcastle.edu.au \\
hebert.perez@gmail.com
\end{tabular}


maximum degrees $\Delta=5,6,9,12$ and 14 , members of this family constitute the largest known graphs of diameter 6 .

Keywords: Degree/diameter problem, Moore bipartite graphs, compounding of graphs.

\section{Introduction}

The topology of a network (such as a telecommunication, multiprocessor, or a local area network, or a social network) is usually modeled by a graph in which vertices represent "nodes" (stations, processors or people) while edges stand for "links" or other types of connections.

When designing networks, several restrictions and/or specifications naturally emerge. Some of the most common limitations lie in the number of connections attached to a node and in the maximum number of links that any node should traverse to reach any other node; see [35]. In graph-theoretical terms, these two parameters represent the degree of a vertex and the diameter of the graph, respectively. These concepts are defined formally in Section 2

The optimality of a network has been interpreted in many distinct ways [35. One possible interpretation can be stated as follows:

An optimal network contains the maximum possible number of nodes, given a limitation on the number of connections attached to a node and a limitation on the number of traversed links between any two farthest nodes.

The preceding interpretation has attracted researchers' attention because of its implications in the design of interconnection networks [35, pp. 91]. Examples of such large topologies are global, regional or local communication networks; computer networks; VLSI circuits; and social networks.

In graph-theoretical terms, this leads to the degree/diameter problem.

Degree/diameter problem: Given natural numbers $\Delta$ and $D$, find the largest possible number of vertices $n_{\Delta, D}$ in a graph of maximum degree $\Delta$ and diameter at most $D$. 
This problem is also known as the $(\Delta, D)$-graph problem. An upper bound on $n_{\Delta, D}$ is given by the Moore bound, see [6, 30].

$$
\begin{aligned}
n_{\Delta, D} \leq & 1+\Delta+\Delta(\Delta-1)+\cdots+\Delta(\Delta-1)^{D-1} \\
& =1+\Delta\left[1+(\Delta-1)+\cdots+(\Delta-1)^{D-1}\right] \\
& = \begin{cases}1+\Delta \frac{(\Delta-1)^{D}-1}{\Delta-2} & \text { if } \Delta>2 \\
2 D+1 & \text { if } \Delta=2\end{cases}
\end{aligned}
$$

The right hand of Equation (1) is known as the Moore bound, and is denoted by $M_{\Delta, D}$. It is well known that graphs attaining this bound exist only for certain special values of the maximum degree and diameter; see [1, 12, 28].

The degree/diameter problem was first studied by Hoffman and Singleton [28] in 1960. However, at present, not much is known about the exact values of $n_{\triangle, D}$. The only known values of $n_{\Delta, D}$ are shown in Table 1

Table 1: Known values of $n_{\Delta, D}$.

\begin{tabular}{||l|l|l||}
\hline \hline$\Delta$ & $D$ & $n_{\Delta, D}$ \\
\hline \hline$\Delta \geq 1$ & $D=1$ & $M_{\Delta, 1}[30]$ \\
\hline$\Delta=2,3,7$ & $D=2$ & $M_{\Delta, 2}[28]$ \\
$\Delta=4,5$ & & $M_{\Delta, 2}-2[18]$ \\
$\Delta=6$ & & $M_{6,2}-5[31]$ \\
\hline$\Delta=2$ & $D=3$ & $M_{2,3}[30]$ \\
$\Delta=3$ & & $M_{3,3}-2[18,[29]$ \\
\hline$\Delta=2$ & $D=4$ & $M_{2,4}[30]$ \\
$\Delta=3$ & & $M_{3,4}-8[8,[11,[1]$ \\
\hline$\Delta=2$ & $D \geq 5$ & $M_{2, D}[30]$ \\
\hline \hline
\end{tabular}

Therefore, research efforts related to the degree/diameter problem cover both proofs of nonexistence of graphs of order close to the Moore bounds and constructions of ever larger graphs; 
one outcome of such constructions is to improve lower bounds on $n_{\Delta, D}$.

Constructions of large graphs were first given in [18]. In that paper, Elspas constructed, among other graphs, the unique graph of degree 4 , diameter 2 and order $M_{4,2}-2$, and the unique graph of degree 5 , diameter 2 and order $M_{5,2}-2$; he also credited Green with obtaining the unique graph of degree 3 , diameter 3 and order $M_{3,3}-2$.

Interest in the degree/diameter problem is further motivated by the accessible on-line table of the largest known graphs for maximum degrees $3 \leq \Delta \leq 16$ and diameters $2 \leq D \leq 10$; see [34]. For more information, we refer the interested reader to the survey by Miller and Širán̆ [30] for history, background and development.

Presently, compounding of graphs is one of the most frequently used techniques for the construction of large graphs of given maximum degree and diameter. The compounding technique was introduced by Bermond, Delorme and Quisquater [4]. It has been used successfully to obtain large graphs, especially in ad hoc combinations with other methods; see, for instance, $[2,3,19,10,13-15,20,22,27,33$.

In [33] Quisquater proposed a compounding method which replaces a single vertex from a Moore bipartite graph with a suitable complete graph. However, in a Moore bipartite graph, it is possible to replace several vertices with copies of suitable complete graphs without increasing the diameter of the bipartite Moore graph. This idea is a result of evolving work started by Gómez, Fiol and Serra [25] in 1993. Subsequently, using Moore bipartite graphs of diameter 6, some improvements in this approach have been put forward in [10, 27], and recently in [23].

In this paper, the technique of graph compounding is used to produce a new family of graphs of maximum degree $\Delta$ and diameter 6 . We extend the approach mentioned in the previous paragraph. By using this extension, we produce new largest known graphs of diameter 6 , which, consequently, improve various entries in the table of the largest known graphs of maximum degree $\Delta$ and diameter $D$, see [34].

The rest of this paper is structured as follows. In Section 2, we present the basic concepts and terminology used throughout this paper. Section 3 gives some useful known properties of Moore bipartite graphs. Section 4 describes the constructive process of the compound graphs 
$H_{\Delta-1}\left(K_{h}\right)$ of diameter 6 , whereas, in Section 5 , we present the members of this family of graphs which constitute the largest known graphs for their respective maximum degrees. Finally in Section 6, we summarize the results obtained.

\section{Basic Definitions}

The terminology and notation used in this section is standard and is consistent with that used in [16].

In this paper $[S]^{k}$ denotes the set of all $k$-subsets of a set $S$; a graph is a pair $G=(V, E)$ of sets satisfying $E \subseteq[V]^{2}$. The elements of $V$ and $E$ are the vertices and edges of the graph $G$, respectively.

The vertex set of a graph $G$ is denoted by $V(G)$, and its edge set by $E(G)$. The number of vertices of $G$ represents the order of $G$, and is denoted by $n(G)$.

For an edge $e=\{x, y\}$, we will write $e=x y$, or simply $x y$. For an edge $e=u v$, we say that $u$ and $v$ are the ends of $e$, adjacent or neighbors, and incident with $e$; also $e$ is incident with $u$ and $v$. Two distinct edges are adjacent if they share an end. The set of neighbors of a vertex $v$ in $G$ is denoted by $N(v)$. We denote by $E(X, Y)$ the set of all edges between elements of a set $X$ and a set $Y$. The degree of a vertex $v$ is the number of edges incident with $v$, and is denoted by $d(v)$. The number $\Delta(G)=\max \{d(v) \mid v \in V\}$ is the maximum degree of $G$.

A set of elements is called independent or stable if it contains no adjacent elements. The cardinality of a set of elements $A$ is denoted by $|A|$.

For $k \geq 0$, a path $P$ is a graph such that

$$
V(P)=\left\{x_{0}, x_{1}, \ldots, x_{k}\right\} \text { and } E(P)=\left\{x_{0} x_{1}, x_{1} x_{2}, \ldots, x_{k-1} x_{k}\right\}
$$

The length of a path is its number of edges. A path of length $k$, denoted by $P_{k}$, is called a $k$-path We will also use the following notation for subpaths of a path $P=x_{0} x_{1} \ldots x_{k}: x_{i} P x_{j}=x_{i} \ldots x_{j}$ where $0 \leq i \leq j \leq k$. 
For $k \geq 3$, a cycle $C$ is a graph such that

$$
V(C)=\left\{x_{0}, x_{1}, \ldots, x_{k-1}\right\} \text { and } E(C)=\left\{x_{0} x_{1}, x_{1} x_{2}, \ldots, x_{k-1} x_{0}\right\}
$$

The length of a cycle $C$ is the number of edges in $C$. A cycle of length $k$, denoted by $C_{k}$, is called a $k$-cycle. The minimum length of a cycle in a graph $G$ is the girth of $G$ and is denoted by $g(G)$. The length of a shortest path from vertex $u$ to vertex $v$ in $G$ represents the distance in $G$ between $u$ and $v$, denoted by $d(u, v)$; if no such path exists, $d(u, v)=\infty$. The diameter of a graph $G$, denoted by $D(G)$, is the largest distance between any two vertices in $G$.

A walk of length $k$ is a sequence of vertices $v_{i}$ and edges $e_{i}, 0 \leq i \leq k$, such that $e_{i}=v_{i} v_{i+1}$, for $i<k$. If $v_{0}=v_{k}$, the walk is closed. We could also refer to a walk by the sequence of its vertices.

Let $G$ and $G^{\prime}$ be two graphs. If $V\left(G^{\prime}\right) \subseteq V(G)$ and $E\left(G^{\prime}\right) \subseteq E(G)$, then $G^{\prime}$ is a subgraph of $G$; denoted by $G^{\prime} \subseteq G$. We define the distance between two subgraphs $H$ and $H^{\prime}$ of a graph $G$ as follows: $d\left(H, H^{\prime}\right)=\min \left\{d(x, y) \mid x \in V(H), y \in V\left(H^{\prime}\right)\right\}$.

Let $G=(V, E)$ be a graph and let $m \geq 2$ be an integer. The graph $G$ is called $m$-partite if it is possible to partition $V$ into $m$ sets (called partite sets) such that no edge joins two vertices in the same set. A 2-partite graph is called bipartite.

A perfect matching between two sets of vertices with the same order $n$ is a set of $n$ independent edges between both sets.

Let $G$ be a bipartite graph with partite sets $V_{1}$ and $V_{2}$ and edge set $E$, denoted by $G=\left(V_{1} \cup V_{2}, E\right)$. A polarity $\pi$ on $G$ is an automorphism of the bipartite graph such that

(i) $\pi\left(V_{1}\right)=V_{2}$ and $\pi\left(V_{2}\right)=V_{1}$.

(ii) $\pi^{2}=i d$,

where $i d$ represents the identity automorphism.

We now define the compounding of graphs. Let $S=\left\{G_{1}, G_{2}, \ldots, G_{k}\right\}$ be a set of graphs. Each element of $S$ is called a source graph and, consequently, $S$ is called the set of source graphs. Let 
$G=(V, E)$ be a graph, called the base graph. In addition, let $\hat{G}=(\hat{V}, \hat{E}=\emptyset)$ be a subgraph of $G$, formed by all those vertices of $G$ which are to be replaced during the compounding process. We will call $\hat{G}$ the replaced graph. Finally, let $f$ be a mapping from $\hat{V}$ to $S$.

The compounding of $S$ into $G$ will be denoted by $G(S)$ or by $G\left(G_{1}, G_{2}, \ldots, G_{k}\right)$. We define it by means of the two following steps:

Step 1: Every vertex $v \in \hat{V}$ is replaced by the graph $f(v) \in S$. The set of added vertices is denoted by $\hat{V}(S)$, that is, $\hat{V}(S)=\bigcup_{v \in \hat{V}} V(f(v))$.

Step 2: The edges incident with $v \in \hat{V}$ are distributed among the vertices of $f(v)$. Note that this step introduces a certain degree of ambiguity, so further specifications will often be needed.

To exemplify this, see Figure 1 , where $S=\left\{C_{3}, C_{4}, K_{4}\right\}$ and the base graph is $K_{4}$. The replaced graph has vertex set $V(\hat{G})=\{a, b, d\}$ and edge set $E(\hat{G})=\emptyset$, and the mapping is $f(a)=C_{3}$, $f(b)=C_{4}$, and $f(d)=K_{4}$.

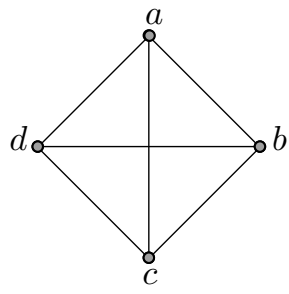

Base graph

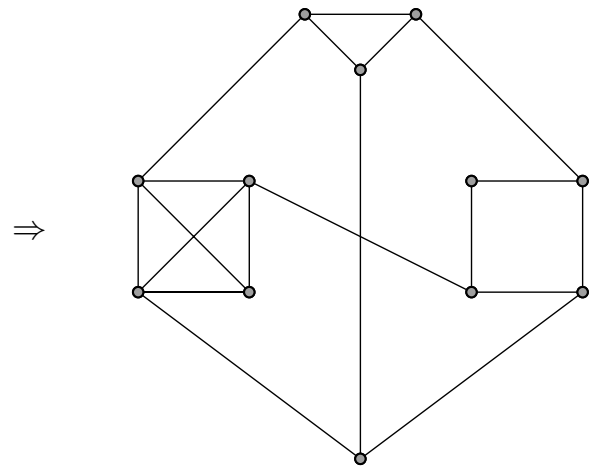

Compound graph

Figure 1: Compound graph.

\section{Moore Bipartite Graphs}

The Moore bipartite bound, i.e. the maximum number $M_{\Delta, D}^{b}$ of vertices in any bipartite graph of maximum degree $\Delta$ and diameter $D$, can be found in Biggs [6]: 


$$
M_{\Delta, D}^{b}= \begin{cases}2 \frac{(\Delta-1)^{D}-1}{\Delta-2} & \text { if } \Delta>2 \\ 2 D & \text { if } \Delta=2\end{cases}
$$

Bipartite graphs attaining this bound are called bipartite Moore graphs. Moore bipartite graphs are $\Delta$-regular graphs and exist only if $D=2,3,4$ or 6 . For $D=2$, they are the complete bipartite graphs of degree $\Delta$. For $D=3,4$ and 6 , bipartite Moore graphs have so far been constructed only if $\Delta-1$ is a prime power [6].

The existence of a Moore bipartite graph of degree $\Delta$ and diameter 3 is equivalent to the existence of a projective plane of order $\Delta-1$ [6]. This graph can be seen as the incidence graph of the corresponding projective plane. We will denote the incidence graph of a projective plane of order $\Delta-1$ by $I_{\Delta-1}$.

A Moore bipartite graph of degree $\Delta$ and diameter 6 will be denoted by $H_{\Delta-1}$.

In any Moore bipartite graph $G$ of degree $\Delta$ and diameter $D$, the following assertions hold; see [21, pp. 85].

1. The girth of $G$ is equal to $2 D$. Therefore, if $x$ and $y$ are two vertices such that $d(x, y)=$ $m<D$, then there exists exactly one $m$-path from $x$ to $y$ in $G$.

2. Given two vertices $x$ and $y$, if $d(x, y)=D$, then there exist $\Delta$ disjoint paths joining $x$ and $y$.

3. Any two vertices are contained in a $2 D$-cycle.

\section{Compound Graphs $H_{\Delta-1}\left(K_{h}\right)$}

The process of construction of these graphs is divided into two parts. The first part is a compounding operation and the second part sets up new adjacency rules.

To describe the graphs, we will use the definition of compounding of graphs presented in Section

2. We base our method on a step-by-step construction showing a sequence of operations required to define these compound graphs. 
Note that the order of any compound graph $G(S)$, using $S=\left\{K_{h}\right\}$ as the set of source graphs, $G$ as the base graph, and $\hat{G}=(\hat{W}, \hat{E}=\emptyset)$ as the replaced graph, is the following.

$$
n(G(S))=n(G)+|\hat{W}|(h-1)
$$

Our compounding operation is slightly different from that described in [10, 23, 27]. We have kept the same notation and terminology in order to achieve a better understanding of the idea. However, conditions to set up the new adjacency rules have been changed.

\section{Part 1: Compounding Operation}

Source Graphs: Given a positive integer $\Delta, G_{1}=K_{h}$, where $K_{h}$ is the complete graph of order $h$, for $h \leq \Delta-1$.

Base Graph: Moore bipartite graph $H_{\Delta-1}$ with partite sets $V$ and $W$, edge set $E$, degree $\Delta$ and diameter 6 . Recall that $\Delta-1$ is a prime power.

Replaced Graph: Let $R$ be the subgraph of $H_{\Delta-1}$ depicted in Figure 2, Let $x$ be a vertex of $V$ and let $N(x)=\left\{x_{0}, x_{1}, \ldots, x_{\Delta-1}\right\}$. We now define the following sets:

1. $W^{0}=N(x)-\left\{x_{\Delta-1}\right\}$.

2. $V^{0}=N\left(W^{0}\right)-\{x\}-\bigcup_{i \in\{0, \ldots, \Delta-2\}}\left\{x_{i \Delta-2}\right\}$.

3. $W^{\prime}=N\left(V^{0}\right)-W^{0}-\bigcup_{\substack{i \in\{0, \ldots, \Delta-2\} \\ j \in\{0, \ldots, \Delta-3\}}}\left\{x_{i j \Delta-2}\right\}$.

The set $W^{\prime} \subset W$ is called the set of replaceable vertices and is highlighted in Figure 2 .

Given a subset $\hat{W}$ of $W^{\prime}$, the replaced graph will then be $\hat{G}=(\hat{W}, \hat{E}=\emptyset)$.

\section{Mapping $f$ :}

$$
f\left(x_{i j k}\right)=K_{h} \quad \text { for all } x_{i j k} \in \hat{W}
$$

Specifying Step 1: Each vertex $x_{i j k}$ of $\hat{W}$ will be replaced by a complete graph $K_{h}$, denoted by $K_{h}^{(i j k)}$, with vertex set $V\left(K_{h}^{(i j k)}\right)=\left\{y_{0}^{(i j k)}, \ldots, y_{h-1}^{(i j k)}\right\}$. 


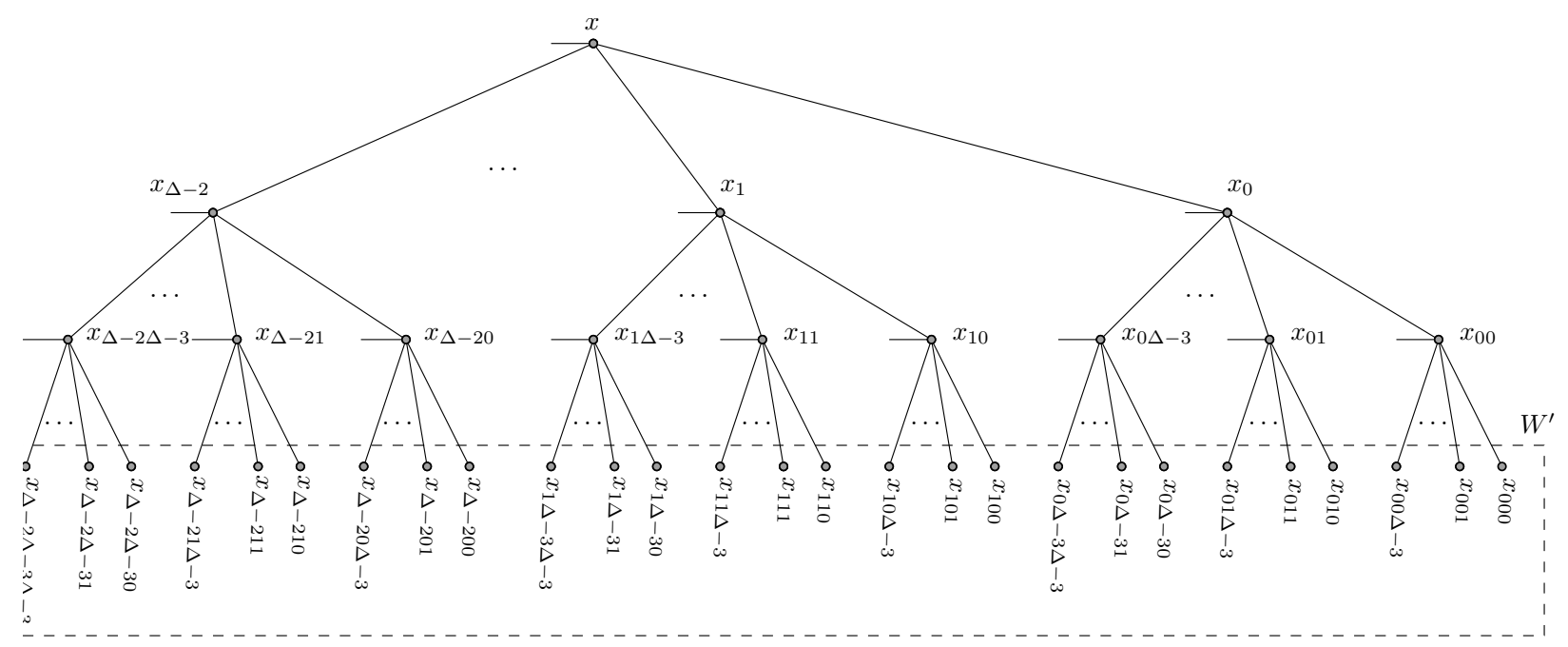

Figure 2: Subgraph $R$ of $H_{\Delta-1}$ to be modified.

The set of added vertices is denoted by $\hat{W}\left(K_{h}\right)$. Therefore, $\hat{W}\left(K_{h}\right)=\bigcup_{x_{i j k} \in \hat{W}} V\left(K_{h}^{(i j k)}\right)$.

Specifying Step 2: The edges incident with each vertex $x_{i j k} \in \hat{W}$ are joined to the vertices of $K_{h}^{(i j k)}$ in such a way that each vertex of $K_{h}^{(i j k)}$ is incident with at least one of these edges. Given a vertex $x_{i j k} \in \hat{W}$ and the complete graph $K_{h}^{(i j k)}$, we denote by $y_{0}^{(i j k)} \in K_{h}^{(i j k)}$ the vertex that has $x_{i j}$ as a neighbor. Then $y_{0}^{(i j k)}$ is connected to at least one other neighbor of $x_{i j k}$.

Resulting Graph: $H_{\Delta-1}^{0}\left(K_{h}\right)$.

Figure 3 illustrates the replacement of a vertex with a triangle by the compounding operation. Note that vertex $y_{0}^{(000)}$ is connected to $x_{00}$ and to another neighbor of $x_{000}$.

\section{Part 2: Introduction of New Edges}

In order to guarantee that the graphs $H_{\Delta-1}\left(K_{h}\right)$ keep diameter 6 , new edges are introduced. The set of edges to be added in this part is denoted by $\hat{E}\left(K_{h}\right)$. Therefore, we define the graph $\hat{G}\left(K_{h}\right)=\left(\hat{W}\left(K_{h}\right), \hat{E}\left(K_{h}\right)\right)$.

We need to introduce new notation and terms. We are now interested in defining the replaced graph $\hat{G}=(\hat{W}, \hat{E})$ completely, that is, to define its edge set. The edge set can be defined as 


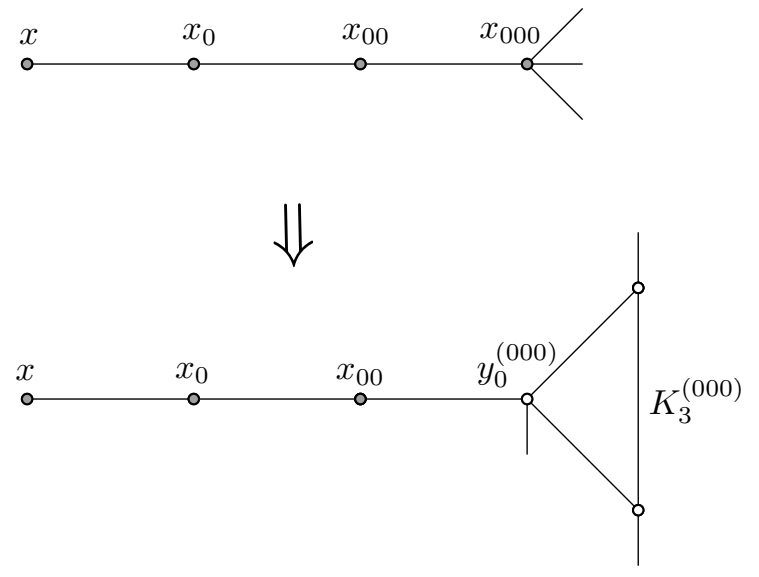

Figure 3: In $H_{3}$, the replacement of vertex $x_{000}$ with $K_{3}$.

follows. For $x_{i j k}, x_{r s t} \in \hat{W} ;\left\{x_{i j k}, x_{r s t}\right\} \in \hat{E} \leftrightarrow \exists \alpha, \beta \in\{0, \ldots, h-1\}$ such that $\left\{y_{\alpha}^{(i j k)}, y_{\beta}^{(r s t)}\right\} \in$ $E\left(V\left(K_{h}^{(i j k)}\right), V\left(K_{h}^{(r s t)}\right)\right)$, that is, $\left\{y_{\alpha}^{(i j k)}, y_{\beta}^{(r s t)}\right\} \in \hat{E}\left(K_{h}\right)$. Figure 4 depicts a graph $\hat{G}\left(K_{3}\right)$ and its corresponding graph $\hat{G}$.

We will call a block any set formed by copies of $K_{h}^{(i j k)}$. However, we will mainly use blocks obtained as copies of $K_{h}^{(i j k)}$, for a fixed $i$. The total number of blocks is denoted by $N_{b}$.

Finally, the margin, denoted by $M$, is the number of edges that can be added to the vertices of each $K_{h}$ so that the maximum degree of $H_{\Delta-1}\left(K_{h}\right)$ continues being at most $\Delta$. It is easy to see that $M=(\Delta-h)(h-1)[27]$.

Now we will describe the introduction of new edges in our approach.

Origin Graph: $H_{\Delta-1}^{0}\left(K_{h}\right)$.

Condition 1: There should be one edge between each pair of copies $K_{h}^{(i j k)}$ and $K_{h}^{(i j t)}$, for $k \neq t$. The maximum number of edges joining a copy $K_{h}^{(i j k)}$ to any other copy $K_{h}^{(i j t)}$, for $k \neq t$, is denoted by $\delta$.

Condition 2: The distance between any two copies of $K_{h}^{(i j k)}$ inside a block should be at most 3. In particular, the distance between any two copies $K_{h}^{(i j k)}$ and $K_{h}^{(i s t)}$ should be at most 3 . 


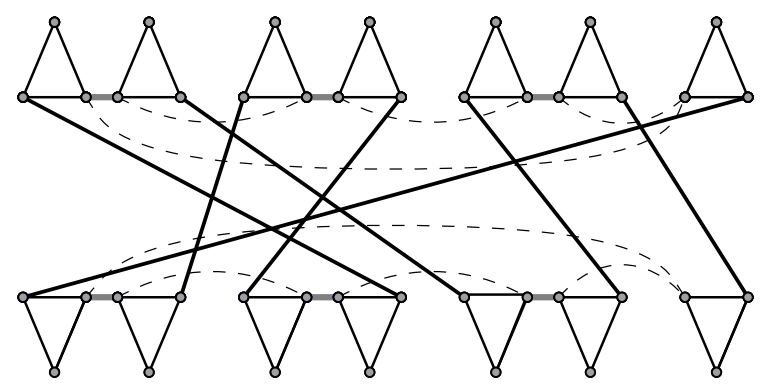

$(a)$

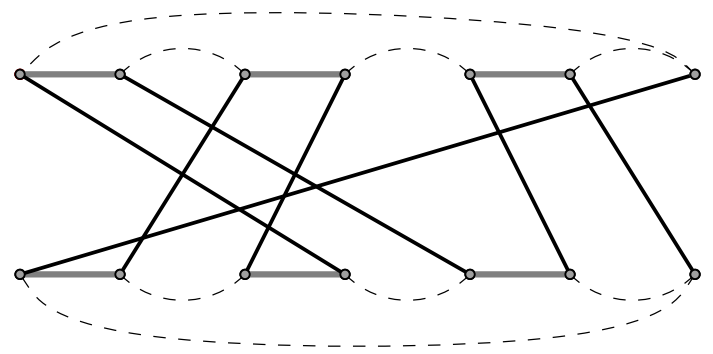

(b)

Figure 4: $(a)$ : Graph $\hat{G}\left(K_{3}\right)$ and $(b)$ : graph $\hat{G}$ obtained from $\hat{G}\left(K_{3}\right)$.

Condition 3: The distance between any two copies $K_{h}^{(i j k)}$ and $K_{h}^{(r s t)}$ should be at most 4 , for $i \neq r$. Equivalently, the distance between any two copies of $K_{h}^{(i j k)}$ from different blocks should be at most 4 .

Condition 3a: The distance between any vertex $y_{\alpha}^{(i j k)}$ and any copy of type $K_{h}^{(r s t)}, i \neq r$, should be at most 5 .

Condition 4: All these adjacencies are added in such a way that the maximum degree of $H_{\Delta-1}\left(K_{h}\right)$ is at most $\Delta$.

Resulting Graph: $H_{\Delta-1}\left(K_{h}\right)$.

Note that either Condition 3 or $3 \mathrm{a}$ can be used to show that the distance between any vertex $y_{\alpha}^{(i j k)} \in K_{h}^{(i j k)}$ and any vertex $y_{\beta}^{(r s t)} \in K_{h}^{(r s t)}, i \neq r$, is at most 6 . Moreover, the cardinality of each block need not be equal.

Figure 5 depicts a block and its intraconnections which satisfy Conditions 1 and 2, while Figure 6 depicts two blocks and their intra and interconnections which satisfy Conditions 1, 2 and 3. 


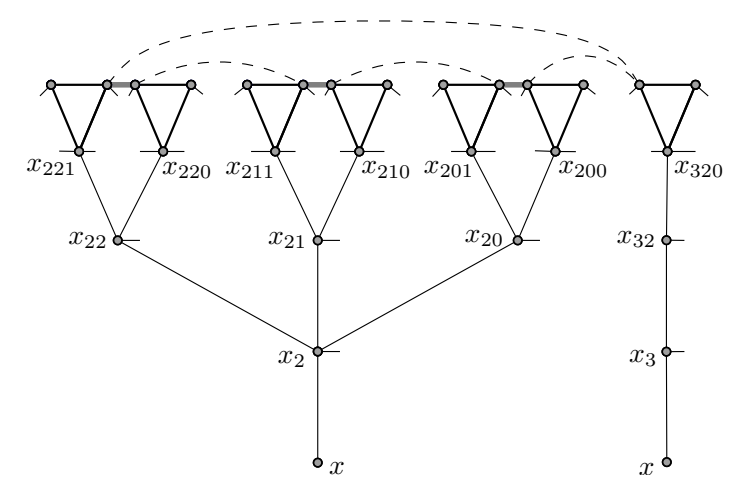

Figure 5: Block $\left\{K_{3}^{(200)}, K_{3}^{(201)}, K_{3}^{(210)}, K_{3}^{(211)}, K_{3}^{(220)}, K_{3}^{(221)}, K_{3}^{(320)}\right\}$ and its intraconnections. Edges guaranteeing Condition 1 are colored in gray and edges guaranteeing Condition 2 are highlighted by a dashed line.

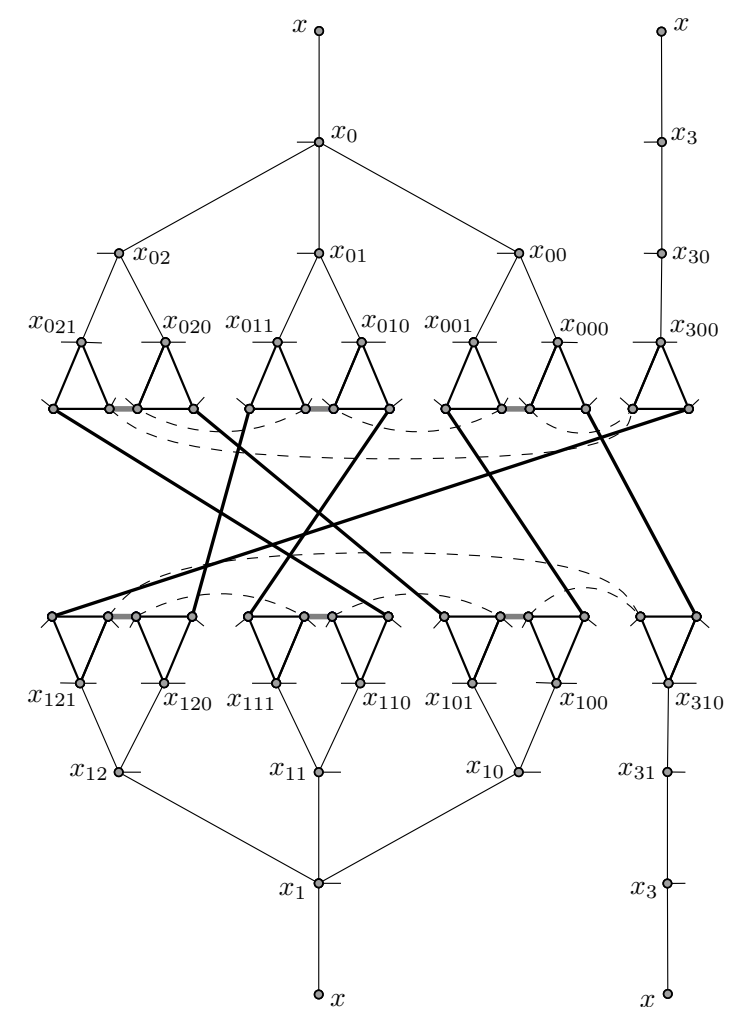

Figure 6: Two blocks, namely, $\left\{K_{3}^{(000)}, K_{3}^{(001)}, K_{3}^{(010)}, K_{3}^{(011)}, K_{3}^{(020)}, K_{3}^{(021)}, K_{3}^{(300)}\right\}$ and $\left\{K_{3}^{(100)}, K_{3}^{(101)}, K_{3}^{(110)}, K_{3}^{(111)}, K_{3}^{(120)}, K_{3}^{(121)}, K_{3}^{(310)}\right\}$, and their interconnections. Edges guaranteeing Condition 1 are colored in gray, and edges guaranteeing Conditions 2 and 3 are highlighted by dashed and heavier lines, respectively. 
Lemma 4.1 In $H_{\Delta-1}\left(K_{h}\right)$, the graph $\hat{G}\left(K_{h}\right)=\left(\hat{W}\left(K_{h}\right), \hat{E}\left(K_{h}\right)\right)$ has diameter 6 .

Proof. Note that, because of Condition 2, in the graph $\hat{G}\left(K_{h}\right)$, the distance between any two copies of $K_{h}^{(i j k)}$ inside a block is 3 . Therefore, the distance between any two vertices $y_{\alpha}^{(i j k)}$ and $y_{\beta}^{(r s t)}$ from the same block is at most 5 . Besides, because of Condition 3 or $3 \mathrm{a}$, the distance between any two vertices $y_{\alpha}^{(i j k)}$ and $y_{\beta}^{(r s t)}$ from different blocks is at most 6 . Thus, the lemma follows from the fact that $\hat{G}\left(K_{h}\right)$ is the union of the $N_{b}$ blocks.

Theorem 4.1 Any graph $H_{\Delta-1}\left(K_{h}\right)$ constructed by following Conditions 1-4 has maximum degree $\Delta$ and diameter 6 .

Proof. Let $H_{\Delta-1}\left(K_{h}\right)$ be a graph satisfying Conditions 1-4. In such a graph, a path not containing vertices of $\hat{W}$ is unaffected.

The maximum number of replaced vertices that can be in a shortest path of any $H_{\Delta-1}\left(K_{h}\right)$ is 2; see the selected subgraph $R$ of $H_{\Delta-1}$ (Figure 2), and recall that the girth of $H_{\Delta-1}$ is 12 . Consequently, the distance between any two vertices will increase by at most two units.

By Lemma 4.1 , the distance between any two vertices $y_{\alpha}^{(i j k)}$ and $y_{\beta}^{(r s t)}$ is at most 6 .

Let us consider two unreplaced vertices $z$ and $t$ at distance 5 in $H_{\Delta-1}$, such that a shortest path $P$ that joins them has two replaced vertices, say $P=z x_{i j k} x_{i j} x_{i j l} r t$. According to Condition 1 , there should exist an edge between each pair of copies $K_{h}^{(i j k)}$ and $K_{h}^{(i j l)}$, say $\left\{y_{\alpha}^{(i j k)}, y_{\beta}^{(i j l)}\right\} \in$ $E\left(V\left(K_{h}^{(i j k)}\right), V\left(K_{h}^{(i j l)}\right)\right)$. Then $z y_{\theta}^{(i j k)} y_{\alpha}^{(i j k)} y_{\beta}^{(i j l)} y_{\gamma}^{(i j l)} r t$ is a path of length at most 6 between $z$ and $t$. This case is exemplified in Figure 7 .

Let us now consider two vertices $z$ and $x_{i j k}$ at distance 5 in $H_{\Delta-1}$, such that $z \in V$ and $x_{i j k} \in \hat{W}$. Let $P=z x_{i m n} x_{i m} x_{i} x_{i j} x_{i j k}$ be a shortest path joining $z$ and $x_{i j k}$. According to Condition 2, there should exist a path of length at most 3 between each pair of copies $K_{h}^{(i m n)}$ and $K_{h}^{(i j k)}$. Then there exists a path of length at most 5 between any pair of vertices $y_{\alpha}^{(i m n)}$ and $y_{\beta}^{(i j k)}$. Thus the distance between the vertex $z$ and vertices $y_{\alpha}^{(i j k)} \in K_{h}^{(i j k)}$ is at most 6 .

Suppose that we have two unreplaced vertices $z$ and $t$ at distance 6 in $H_{\Delta-1}$. Then, as there are $\Delta$ disjoint paths between them, there exists a 6 -path between $z$ and $t$ unaffected by the replacements. 
Finally, consider two vertices $z$ and $x_{i j k}$ at distance 6 in $H_{\Delta-1}$, such that $z \notin \hat{W}$ and $x_{i j k} \in \hat{W}$. Then there exist $\Delta$ disjoint paths between them. We need to check that the distance between the vertex $z$ and vertices $y_{\alpha}^{(i j k)} \in K_{h}^{(i j k)}$ is at most 6 .

Because of the structure of the selected subgraph $R$ of $H_{\Delta-1}$ and the fact that $g\left(H_{\Delta-1}\right)=12$, a shortest path linking $x_{i j k}$ to another replaced vertex passes through $x_{i j}$. Therefore, in $H_{\Delta-1}$, there is a unique shortest path $P_{6}$ joining $x_{i j k}$ to $z$ with one further replaced vertex; the remaining $\Delta-1$ paths, namely, $P_{6}^{1}, \ldots, P_{6}^{\Delta-1}$, have no replaced vertex other than $x_{i j k}$. Thus, there exists a unique vertex $y_{\alpha}^{(i j k)}$ of $K_{h}^{(i j k)}$ contained in $P_{6}^{\prime}=z P_{6} x_{i j} y_{\alpha}^{(i j k)} \in H_{\Delta-1}\left(K_{h}\right)$. Let us define the 6-paths $P_{6}^{i i}=z P_{6}^{i} y_{\theta}^{(i j k)} \in H_{\Delta-1}\left(K_{h}\right)$, for $i=1, \ldots, \Delta-1$, between $z$ and any $y_{\theta}^{(i j k)} \in K_{h}^{(i j k)}$. Then the remaining $h-1$ vertices $y_{\beta}^{(i j k)} \neq y_{\alpha}^{(i j k)}$ of $K_{h}^{(i j k)}$ are connected to $z$ through one of the 6-paths $P_{6}^{\prime i}$, for $i=1, \ldots, \Delta-1$. Let us now consider the vertex $y_{\alpha}^{(i j k)} \in K_{h}^{(i j k)}$ contained in $P_{6}^{\prime}$. Then $y_{\alpha}^{(i j k)}$ is adjacent to $x_{i j}$, and consequently, the vertex $y_{\alpha}^{(i j k)}$ is the vertex $y_{0}^{(i j k)}$. Therefore, due to the applied compounding operation, the vertex $y_{0}^{(i j k)}$ is connected to $x_{i j}$ and to at least another neighbor of $x_{i j k}$. Hence, one of the 6-paths $P_{6}^{\prime i} \in H_{\Delta-1}\left(K_{h}\right)$, for $i=1, \ldots, \Delta-1$, connects $y_{0}^{(i j k)}$ to $z$. This case is exemplified in Figure 8 .

\section{New Largest Known Graphs of Diameter 6}

In order to produce $H_{\Delta-1}\left(K_{h}\right)$ graphs of large order, we need to link the copies of $K_{h}$ according to Conditions 1-4 by following the structure of a certain graph. In previous work under other conditions, for instance in [27], copies of $K_{h}$ were joined according to a special graph of diameter 2, whereas in [23], such copies were joined according to the structure of bipartite graphs of diameter 3 .

In this section we describe the new graphs that allow us to produce largest known graphs of diameter 6 for certain maximum degrees.

We introduce a new parameter $M^{\prime}=M-\delta$ which represents the number of edges that can be added to each copy $K_{h}$ after the application of Condition 1. 

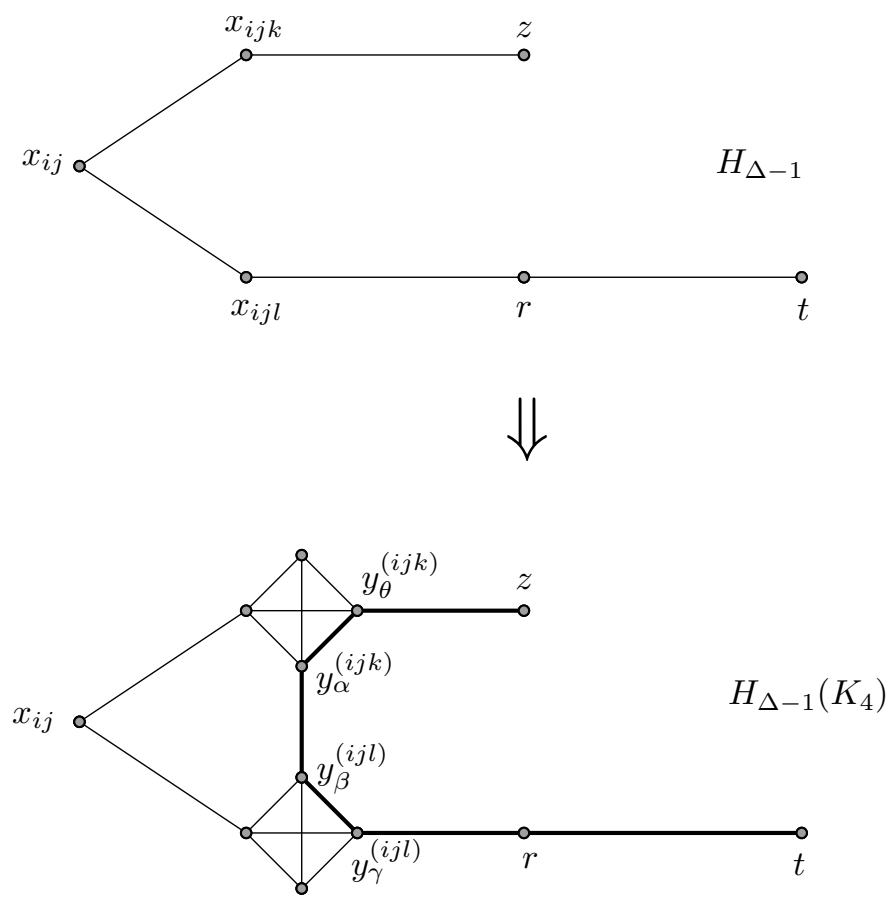

Figure 7: Path of length 6 between $z$ and $t$ in $H_{\Delta-1}\left(K_{4}\right)$, namely, $z y_{\theta}^{(i j k)} y_{\alpha}^{(i j k)} y_{\beta}^{(i j l)} y_{\gamma}^{(i j l)} r t$.
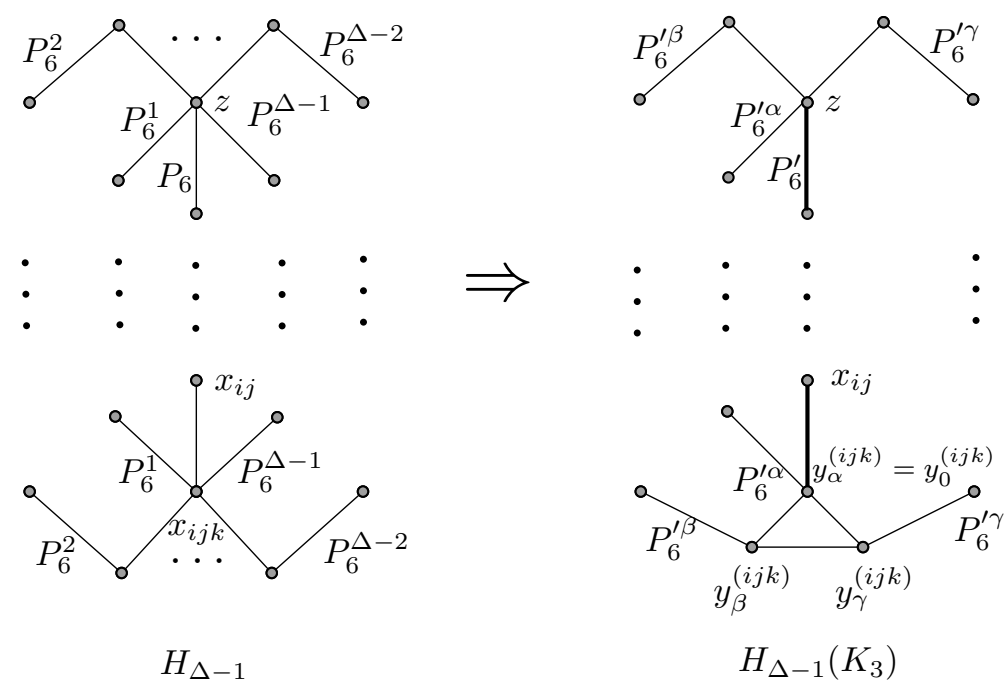

Figure 8: Paths of length 6 between $z$ and vertices $y_{\theta}^{(i j k)} \in K_{3}^{(i j k)}$ in $H_{\Delta-1}\left(K_{3}\right)$. 


\subsection{Constructing $H_{\Delta-1}\left(K_{h}\right)$ Graphs: Approach 1}

In this approach, copies of $K_{h}$ are joined by following the structure of a modified $N_{b}$-partite graph, that is, $\hat{G}$ is a modified $N_{b}$-partite graph. To be more precise, $\hat{G}$ is constructed in the following way. We take $N_{b}$ sets of vertices $\left\{A_{1}, \ldots, A_{N_{b}}\right\}$, join $A_{i}$ to $A_{j}$, for $i \neq j$, by means of a perfect matching, and finally, vertices of each $A_{i}$ are joined by following the structure of a graph of diameter 3 .

\section{Graph $H_{4}\left(K_{3}\right)$ : the New Largest $(5,6)$-Graph}

The graph $H_{4}\left(K_{3}\right)$ is constructed by setting up the following blocks $\left\{K_{3}^{(0 j k)}, K_{3}^{(300)}\right\},\left\{K_{3}^{(1 j k)}, K_{3}^{(310)}\right\}$ and $\left\{K_{3}^{(2 j k)}, K_{3}^{(320)}\right\}$, for $j \in\{0,1,2\}$ and $k \in\{0,1\}$.

We then connect any two blocks as shown in Figure 9 . Note that $\hat{G}$ is formed by three sets of 7 vertices and vertices of the same set are connected by a 7-cycle. This graph $\hat{G}$ is partially represented in Figure $4(b)$.

The graph $H_{4}\left(K_{3}\right)$ so constructed has degree 5 and diameter 6 . After applying the compounding operation to these 21 vertices, $M=(5-3)(3-1)=4$. That means that we can add 4 edges to each $K_{3}$. Furthermore, after applying Condition 1 , we have $\delta=1$, so $M^{\prime}=4-1=3$.

In order to check the fulfillment of Condition 2, note that any two copies $K_{3}^{(i j k)}$ inside a block are joined by a 7 -cycle. Besides, there is a 3 -path between any two copies $K_{3}^{(3 j k)}$. These connections are highlighted by a dashed line; see Figure 9.

Therefore we only need to check that the distance between any two copies $K_{3}^{(i j k)}$ and $K_{3}^{(o p m)}$, for $i \neq o$, is at most 4 ; or, equivalently, that the distance between any two copies of $K_{3}^{(i j k)}$ from different blocks is at most 4 .

First of all, Figure 9 shows that the previous assertion is true for any copy $K_{3}^{(0 j k)}, K_{3}^{(1 m n)}, K_{3}^{(300)}$ and $K_{3}^{(310)}$. This fact can be easily verified by following the connections between such graphs. These connections are highlighted by a heavier line. By way of illustration, we will now show that the distance between $K_{3}^{(000)}$ and graphs $K_{3}^{(1 m n)}$, the graph $K_{3}^{(300)}$, and the graph $K_{3}^{(310)}$, is at most 4 . 
i. $K_{3}^{(000)}$ and $K_{3}^{(300)}, P=y_{2}^{(000)} y_{2}^{(300)}$.

ii. $K_{3}^{(000)}$ and $K_{3}^{(310)}, P=y_{1}^{(000)} y_{1}^{(310)}$.

iii. $K_{3}^{(000)}$ and $K_{3}^{(100)}, P=y_{1}^{(000)} y_{1}^{(310)} y_{2}^{(310)} y_{2}^{(100)}$.

iv. $K_{3}^{(000)}$ and $K_{3}^{(101)}, P=y_{1}^{(000)} y_{1}^{(310)} y_{2}^{(310)} y_{2}^{(100)} y_{2}^{(101)}$.

v. $K_{3}^{(000)}$ and $K_{3}^{(121)}, P=y_{1}^{(000)} y_{1}^{(310)} y_{2}^{(310)} y_{2}^{(121)}$.

vi. $K_{3}^{(000)}$ and $K_{3}^{(120)}, P=y_{1}^{(000)} y_{1}^{(310)} y_{2}^{(310)} y_{2}^{(121)} y_{2}^{(120)}$.

vii. $K_{3}^{(000)}$ and $K_{3}^{(110)}, P=y_{2}^{(000)} y_{2}^{(300)} y_{2}^{(021)} y_{1}^{(021)} y_{1}^{(110)}$.

viii. $K_{3}^{(000)}$ and $K_{3}^{(111)}, P=y_{2}^{(000)} y_{2}^{(001)} y_{2}^{(010)} y_{1}^{(010)} y_{1}^{(111)}$.

In the graph $H_{4}\left(K_{3}\right)$, vertices $y_{1}^{(i j k)}$ and $y_{1}^{(o p m)}$ link any two blocks (Figure 9 shows only a part of these connections). Note that each of these vertices has two connections for this purpose, and only one of these connections is needed for establishing a path of length at most 4 between any two copies $K_{3}^{(i j k)}$ and $K_{3}^{(o p m)}$ from two different blocks.

Following Equation (3), we obtain that the order of the new graph $H_{4}\left(K_{3}\right)$ is $n\left(H_{4}\right)+21 \times 2$, that is, 2772. This is an improvement on the order of the previous largest known graph of maximum degree 5 and diameter 6 , which had 2766 vertices.

\section{Graph $H_{5}\left(K_{4}\right)$ : the New Largest $(6,6)$-Graph}

For the graph $H_{5}\left(K_{4}\right)$, we set up the blocks as follows: $\left\{K_{4}^{(0 j k)}, K_{4}^{(030)}\right\},\left\{K_{4}^{(1 j k)}, K_{4}^{(130)}\right\}$, $\left\{K_{4}^{(2 j k)}, K_{4}^{(230)}\right\},\left\{K_{4}^{(3 j k)}, K_{4}^{(330)}\right\}$ and $\left\{K_{4}^{(4 j k)}, K_{4}^{(430)}\right\}$, for $j \in\{0,1,2\}$ and $k \in\{0,1\}$.

As before, we connect any two blocks as shown in Figure 10 . Note that, in this case, $\hat{G}$ is formed by five sets of 7 vertices and vertices of the same set are connected by a 7 -cycle.

The graph $H_{5}\left(K_{4}\right)$ so constructed has degree 6 and diameter 6 . After applying the compounding operation to these 35 vertices, $M=(6-4)(4-1)=6$, we can add 6 edges to each $K_{4}$. Furthermore, after applying Condition 1 , we have $\delta=1$, so $M^{\prime}=6-1=5$. 


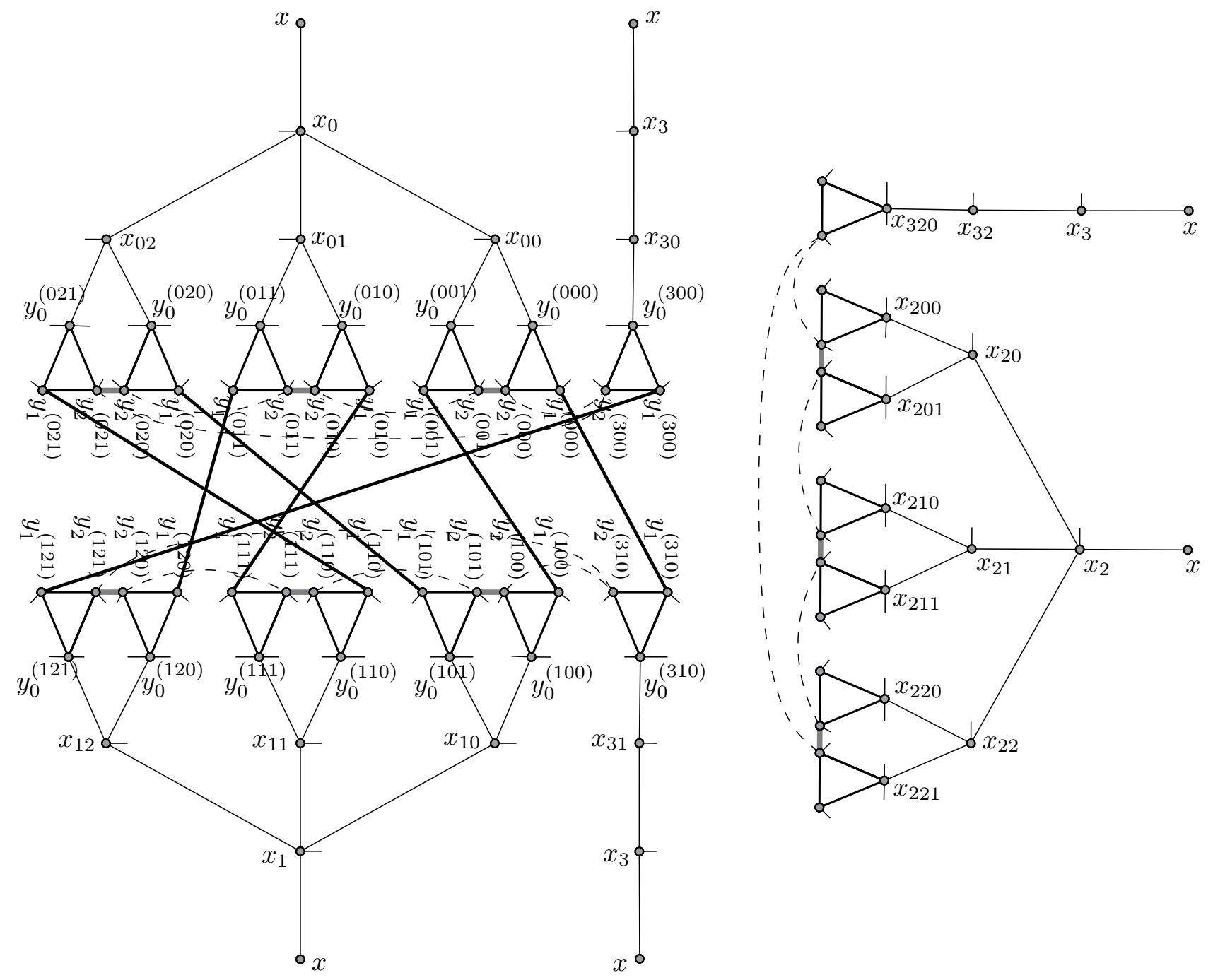

Figure 9: Basic configuration of the $H_{4}\left(K_{3}\right)$ graph of order 2772. 
In Figure 10 connections between blocks are highlighted by a heavier line. Moreover, note that for each copy $K_{4}^{(i j k)}$ of a block, there are exactly two vertices (shown in white; see Figure 10 with two connections each, in order to connect two different blocks, and only one of these connections is needed for establishing a path of length at most 4 between any two copies $K_{4}^{(i j k)}$ and $K_{4}^{(o p m)}$ from two different blocks.

Following Equation (3), we obtain that the order of the new graph $H_{5}\left(K_{4}\right)$ is $n\left(H_{5}\right)+35 \times 3$, that is, 7917. This is an improvement on the order of the previous largest known graph of maximum degree 6 and diameter 6 , which had 7908 vertices.

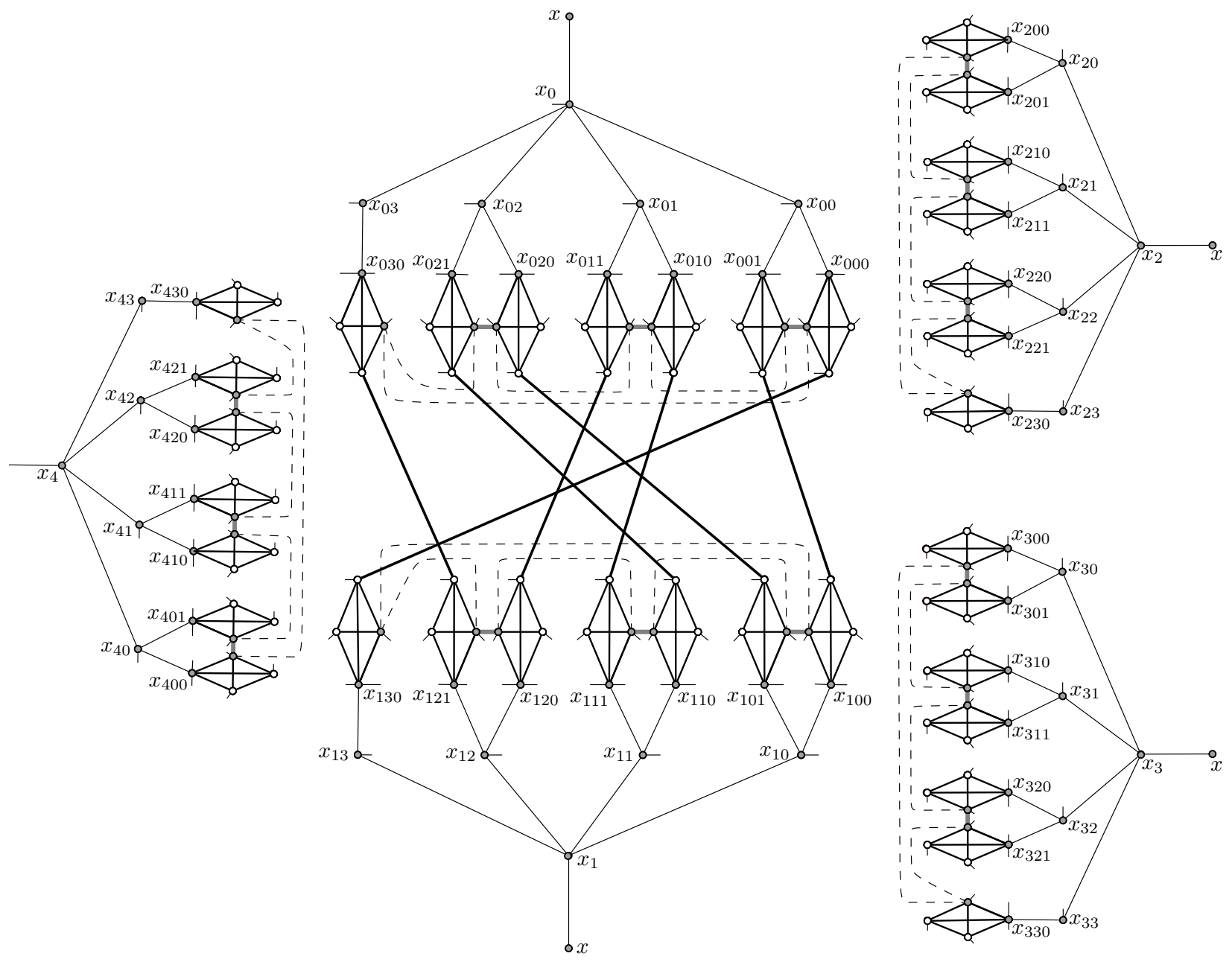

Figure 10: Basic configuration of the $H_{5}\left(K_{4}\right)$ graph of order 7917. 


\subsection{Constructing $H_{\Delta-1}\left(K_{h}\right)$ Graphs: Approach 2}

In this second approach, copies of $K_{h}$ are joined by following the structure of a compound graph of diameter 3 , denoted $G\left(B_{\pi}\right)$, that is, $\hat{G}$ is isomorphic to $G\left(B_{\pi}\right)$. We define graphs $G\left(B_{\pi}\right)$ in the next subsection.

\subsubsection{Graphs $G\left(B_{\pi}\right)$}

Graphs $G\left(B_{\pi}\right)$ are constructed as follows. Take a graph $G$ and a bipartite graph $B_{\pi}=\left(V_{1} \cup V_{2}, E\right)$. Let us assume that $B_{\pi}$ has a polarity $\pi$. As $B_{\pi}$ has a polarity, we make no distinction between its two partite sets, that is $V_{1}=V_{2}=V$. Now we make the compounding of $B_{\pi}$ into $G$. To be more precise, we replace each vertex $u$ in $G$ with $S_{u}=V$. If $u$ and $v$ are adjacent in $G, S_{u}$ and $S_{v}$ are then connected by following the structure of $B_{\pi}$. We denote a vertex $t$ of $G\left(B_{\pi}\right)$ such that $t \in S_{u}$ by the pair $\left(t, S_{u}\right)$. Note that we can match the vertex $\left(t, S_{u}\right)$ in $G\left(B_{\pi}\right)$ with the vertex $t$ of $V$ in $B_{\pi}$. Depending on our particular needs, we can assume that $t$ belongs to $V_{1}$ or $V_{2}$. Graphs $G\left(B_{\pi}\right)$ are exemplified in Figure 11 .

Some properties of graphs $G\left(B_{\pi}\right)$ are shown in the next theorem. We first recall some known properties of bipartite graphs:

If $B=\left(V_{1} \cup V_{2}, E\right)$ is any bipartite graph of even (odd) diameter $D_{B}$ then the distance between $x \in V_{1}$ and any $y \in V_{2}\left(y \in V_{1}\right)$ is at most $D_{B}-1$.

(i) Consequently, from any vertex of $V_{i}$, for $i=1,2$, we reach all vertices from either $V_{1}$ or $V_{2}$ by means of paths of length at most $D_{B}-1$.

(ii) Moreover, if we have two vertices $u_{0}$ and $u_{l}$ joined by a path $P$ of length $l=D_{B}-1-2 r$, for $r \geq 0$, say $P=u_{0} u_{1} \ldots u_{l}$, we can find a walk $Z$ of length $D_{B}-1$ between $u_{0}$ and $u_{l}$. In fact, $Z=Z^{\prime} \cup P$, where $Z^{\prime}$ is any closed walk of length $2 r$, starting at $u_{0}$. Note that we can always obtain such a walk $Z^{\prime}$, for instance, we can take $Z^{\prime}=\underbrace{u_{0} u_{1} u_{0} u_{1} \ldots u_{1} u_{0}}_{2 r \text { steps }}$.

Theorem 5.1 The graphs $G\left(B_{\pi}\right)$ have maximum degree $\Delta(G) \times \Delta\left(B_{\pi}\right)$, order $\frac{n(G) n\left(B_{\pi}\right)}{2}$ and diameter $\max \left\{D(G), D\left(B_{\pi}\right)\right\}$. 

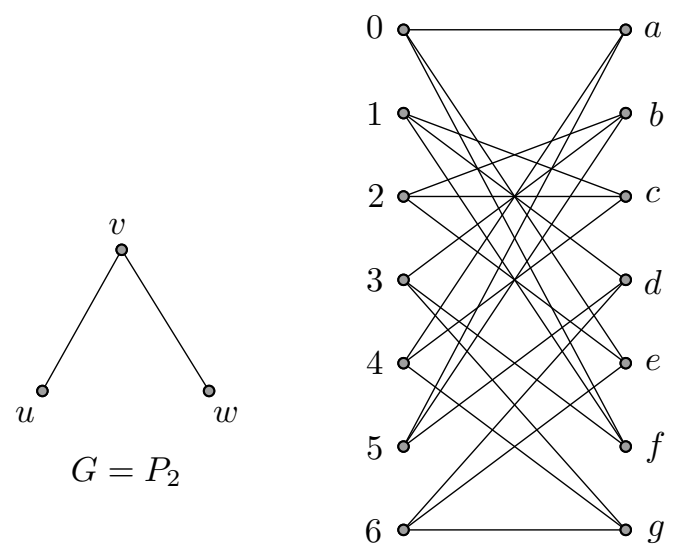

$$
\begin{aligned}
& \pi(0)=a \\
& \pi(1)=b \\
& \pi(2)=c \\
& \pi(3)=d \\
& \pi(4)=e \\
& \pi(5)=f \\
& \pi(6)=g
\end{aligned}
$$

$B_{\pi}=$ Heawood graph $=I_{2} \quad$ a polarity $\pi$ in $B_{\pi}$

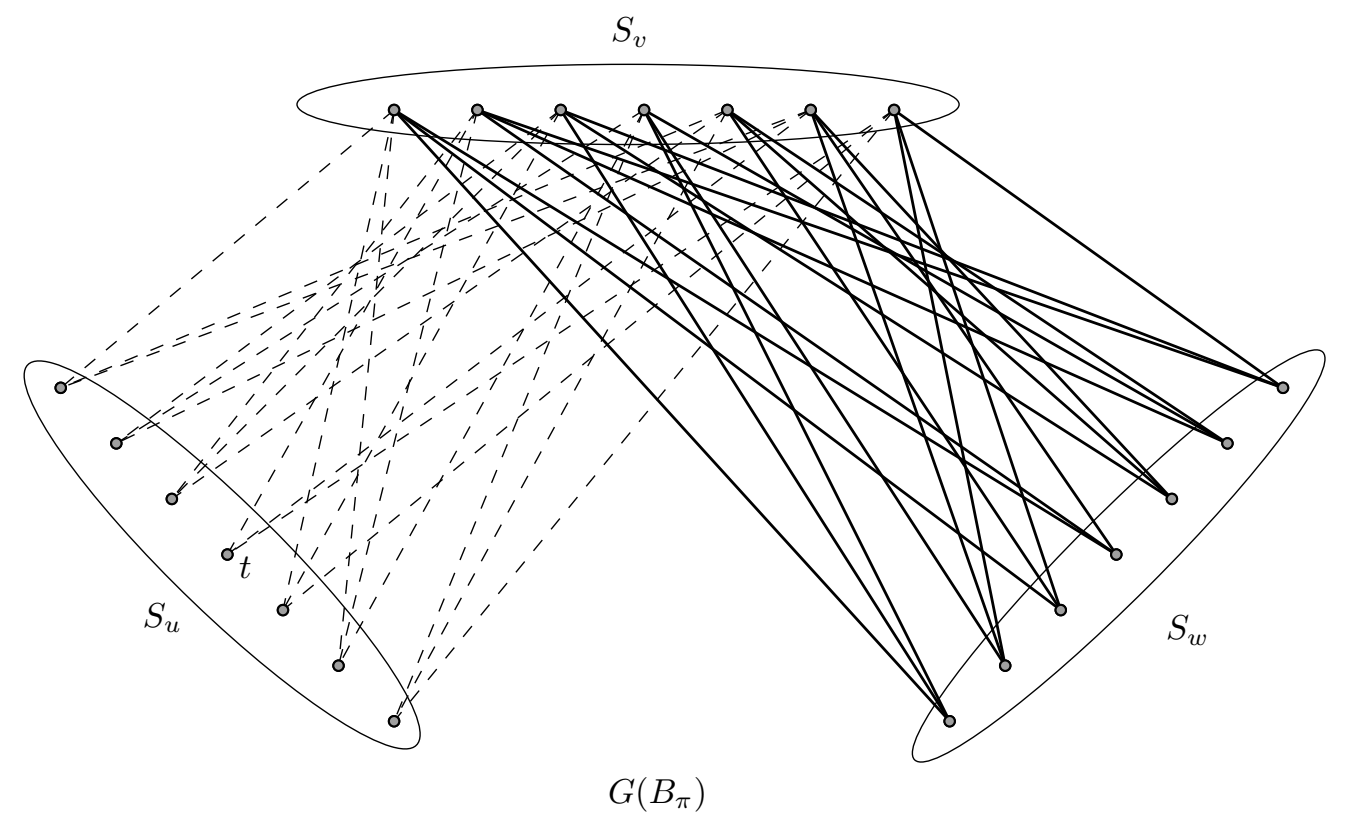

Figure 11: Example of a $G\left(B_{\pi}\right)$ graph; $G=P_{2}$ and $B_{\pi}=$ Heawood graph. 
Proof. The assertion about the maximum degree and the order follows from the construction, so we concentrate on proving the assertion regarding the diameter.

Let $z_{0}$ and $z_{k}$ be vertices of $G$ at distance $k$ and let $P=z_{0} z_{1} \ldots z_{k}$ be a shortest path joining them. Furthermore, let $u$ and $v$ be vertices of $G\left(B_{\pi}\right)$, such that $u \in S_{z_{0}}$ and $v \in S_{z_{k}}$; see Figure 12

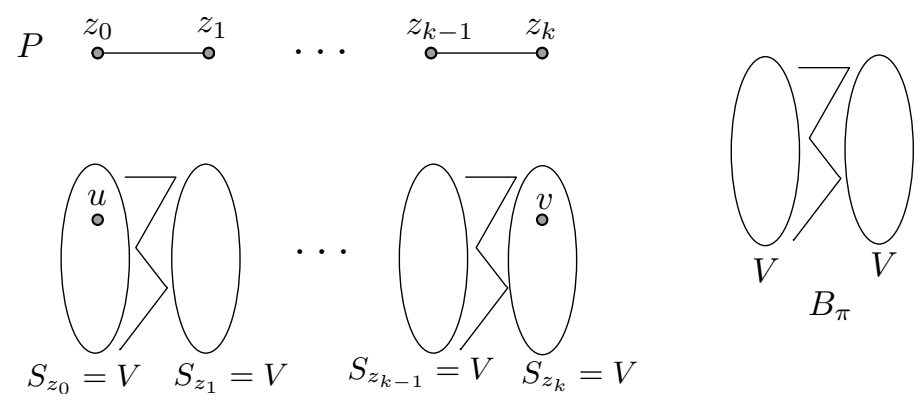

Figure 12: Path of length $k$ in $G$ and the corresponding structure in $G\left(B_{\pi}\right)$.

Our aim is to prove that, given any two vertices $u$ and $v$ in $G\left(B_{\pi}\right), d_{G\left(B_{\pi}\right)}(u, v)=\max \left\{k, D\left(B_{\pi}\right)\right\}$. More precisely, we take a path $P$ of length $k$ between two vertices $z_{0}$ and $z_{k}$ in $G$, say $P=$ $z_{0} z_{1} \ldots z_{k}$, and then we prove that $d_{G\left(B_{\pi}\right)}\left(\left(u, S_{z_{0}}\right),\left(v, S_{z_{k}}\right)\right)=\max \left\{k, D\left(B_{\pi}\right)\right\}$.

Since $B_{\pi}=\left(V_{1} \cup V_{2}, E\right)$ has a polarity, we can match $\left(u, S_{z_{0}}\right)$ with a vertex $u$ in $V_{1}$.

When $k=0$ or $1, u$ and $v$ are located in $B_{\pi}$, so $d_{G\left(B_{\pi}\right)}(u, v)=d_{B_{\pi}}(u, v)$. Henceforth, $k>1$.

We distinguish two cases.

Case 1. $k \geq D\left(B_{\pi}\right)$.

Let $u_{l}$ be any vertex different from $u$ in $V_{1}\left(V_{2}\right)$, provided that $D\left(B_{\pi}\right)$ is odd (even). Let $Q$ be a shortest path in $B_{\pi}$ between $u$ and $u_{l}$, say $Q=u u_{1} \ldots u_{l}$. Because of $(i)$, we may assume that the length of $Q$ has the form $l=D\left(B_{\pi}\right)-1-2 r$, for $r \geq 0$.

By $(i i)$, we construct a walk $Z$ of length $D\left(B_{\pi}\right)-1$ between $u$ and $u_{l}$ in $B_{\pi}$. Let us take $Z=\underbrace{u u_{1} u \ldots u}_{2 r \text { steps }} u_{1} u_{2} \ldots u_{l}$.

Let us now consider the vertex $\left(u_{l}, S_{D\left(B_{\pi}\right)-1}\right)$. 
Since $B_{\pi}$ has a polarity and by using $(i)$, from $\left(u, S_{z_{0}}\right)$ we reach $\left(u_{l}, S_{z_{D\left(B_{\pi}\right)-1}}\right)$ by means of the $\left(D\left(B_{\pi}\right)-1\right)$-path $\left(u, S_{z_{0}}\right)\left(u_{1}, S_{z_{1}}\right)\left(u, S_{z_{2}}\right) \ldots\left(u, S_{z_{2 r}}\right)\left(u_{1}, S_{z_{2 r+1}}\right) \ldots\left(u_{l}, S_{z_{D(B \pi}-1}\right)$.

Thus, as there are $\left(k-\left(D\left(B_{\pi}\right)-1\right)\right)$-paths from $\left(v, S_{z_{k}}\right)$ to certain vertices in $S_{z_{D\left(B_{\pi}\right)-1}}$, we have just proved that there is a path of length $k$ between $\left(u, S_{z_{0}}\right)$ and $\left(v, S_{z_{k}}\right)$; see Figure 13
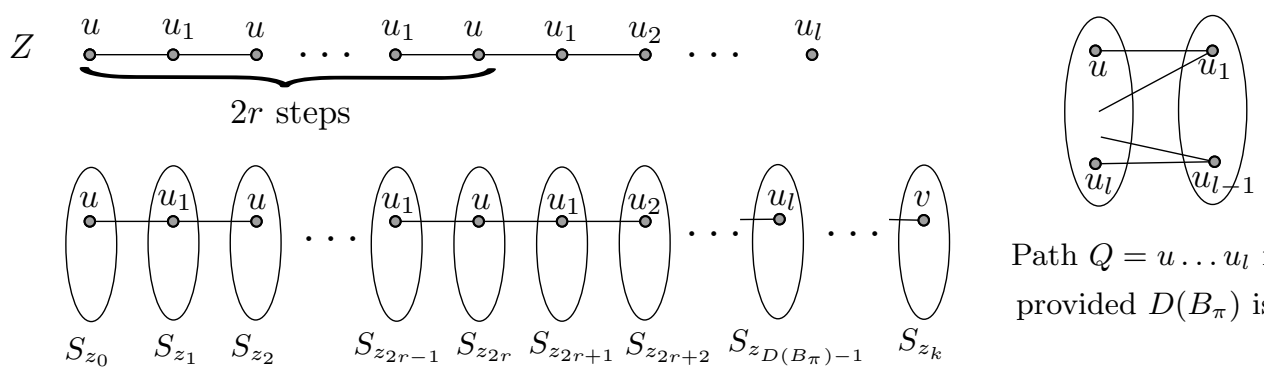

Path $Q=u \ldots u_{l}$ in $B_{\pi}$, provided $D\left(B_{\pi}\right)$ is odd

Figure 13: Path of length $k$ between $\left(u, S_{z_{0}}\right)$ and $\left(v, S_{z_{k}}\right)$ in $G\left(B_{\pi}\right)$.

Case 2. $k<D\left(B_{\pi}\right)$.

In this case we consider two additional subcases.

Case $2.1 k+L_{1}=D\left(B_{\pi}\right)-1$, where $L_{1}$ is even.

As before, we can associate $\left(v, S_{z_{k}}\right)$ with a vertex $v \neq u$ in $V_{1}\left(V_{2}\right)$, provided that $D\left(B_{\pi}\right)$ is odd (even). Therefore, we can take $Q$ as a path of length $k=D\left(B_{\pi}\right)-1-L_{1}$ in $B_{\pi}$ joining $u$ and $v$, say $Q=u u_{1} u_{2} \ldots u_{k-1} v$, for $L_{1} \geq 0$.

Now the vertices $\left(u, S_{z_{0}}\right)$ and $\left(v, S_{z_{k}}\right)$ are joined by the $k$-path $\left(u, S_{z_{0}}\right)\left(u_{1}, S_{z_{1}}\right)\left(u_{2}, S_{z_{2}}\right) \ldots$

$\ldots\left(u_{k-1}, S_{z_{k-1}}\right)\left(v, S_{z_{k}}\right)$.

Case $2.1 k+L_{1}=D\left(B_{\pi}\right)-1$, where $L_{1}$ is odd.

As $k>1$, this subcase can be reduced to the above subcase. In fact, $k-1+L_{2}=D\left(B_{\pi}\right)-1$, where $L_{2}=L_{1}+1$. Therefore, we are able to reach any vertex in $S_{z_{k-1}}$ in at most $D\left(B_{\pi}\right)-1$ steps. Thus, as there are edges from $\left(v, S_{z_{k}}\right)$ to certain vertices in $S_{z_{k-1}}$, there is a path from $\left(u, S_{z_{0}}\right)$ to $\left(v, S_{z_{k}}\right)$ of length at most $D\left(B_{\pi}\right)$.

Hence, in Case $2, d_{G\left(B_{\pi}\right)}\left(\left(u, S_{z_{0}}\right),\left(v, S_{z_{k}}\right)\right) \leq D\left(B_{\pi}\right)$. 


\subsubsection{Construction of Graphs $H_{8}\left(K_{6}\right), H_{11}\left(K_{8}\right)$ and $H_{13}\left(K_{10}\right)$}

Having defined graphs $G\left(B_{\pi}\right)$ and deduced some of their properties, we are now ready to present the constructions of other largest known graphs $H_{\Delta-1}\left(K_{h}\right)$.

General Construction: Let us take a graph $G$ and a bipartite graph $B_{\pi}$ with a polarity $\pi$; both graphs have diameter 3 .

Step 1: We label the vertices of $G$ in such a way that two vertices have the same label only if they are at distance 2 in $G$. A vertex in $G$ can have several labels; for instance if $d(x, y)=d(y, z)=2$ and $d(x, z)=4$, then the labels of vertices $x, y$ and $z$ could be $l_{1},\left\{l_{1}, l_{2}\right\}$ and $l_{2}$, respectively.

Step 2: We construct the graph $G\left(B_{\pi}\right)$. Each vertex $\left(u, S_{z_{i}}\right) \in G\left(B_{\pi}\right)$ takes only one of the labels from the vertex $z_{i} \in G$, so we now denote the vertex $\left(u, S_{z_{i}}\right)$ of $G\left(B_{\pi}\right)$ by the triple $\left(u, S_{z_{i}}, l\right)$, where $l$ is one of the labels of $z_{i} \in G$. The distribution of these labels in each $S_{z_{i}}$ is done according to the specific case. The graph $\hat{G}$ is isomorphic to $G\left(B_{\pi}\right)$ and keeps the same labeling.

Step 3: In $\hat{G}\left(K_{h}\right)$, the copy $K_{h}^{(i j k)}$ keeps the same label as $x_{i j k}=\left(u, S_{z_{i}}, l\right)$ in $\hat{G}$.

Step 4: In $\hat{G}\left(K_{h}\right)$, a block is formed by copies of $K_{h}^{(i j k)}$, for a fixed $i$ with the same label. The total number of labels equals the total number of blocks $\left(N_{b}\right)$.

Step 5: Condition 1 of Theorem 4.1 is applied.

Theorem 5.2 If a graph $H_{\Delta-1}\left(K_{h}\right)$ obtained from the above construction satisfies the requirements

(i) The cardinality of any block is at most $(\Delta-2)^{2}$. For a fixed $i,(\Delta-2)^{2}$ is the number of vertices $u$ in $W^{\prime}$ such that $d\left(x_{i}, u\right)=2$ (see Figure 2), and

(ii) $M^{\prime}=M-\delta \geq \Delta\left(G\left(B_{\pi}\right)\right)=\Delta(G) \times \Delta\left(B_{\pi}\right)$, and

(iii) $h \leq \Delta\left(B_{\pi}\right)+1$ 
then $H_{\Delta-1}\left(K_{h}\right)$ has maximum degree $\Delta$ and diameter 6 .

Proof. The requirement (ii) guarantees that the maximum degree of $H_{\Delta-1}\left(K_{h}\right)$ is at most $\Delta$. Therefore, we only need to prove that Conditions 2 and 3 of Theorem 4.1 hold; and in those cases, where they are not met, we should prove that the diameter is nevertheless still 6 .

By Step 4, as blocks are formed by copies of $K_{h}$ with the same label, it is guaranteed that these copies are at distance 3 , that is, Condition 2 of Theorem 4.1 holds.

Let $\left(x_{i j k}, S_{z_{\alpha}}\right)$ and $\left(x_{r s t}, S_{z_{\beta}}\right)$ be vertices of $\hat{G}$, such that $d_{\hat{G}}\left(x_{i j k}, x_{r s t}\right)=3$. Then, in $\hat{G}\left(K_{h}\right)$, copies $K_{h}^{(i j k)}$ and $K_{h}^{(r s t)}$ belong to different blocks.

As $D\left(B_{\pi}\right)$ is 3 , vertices in $B_{\pi}$ from the same partite set are at distance at most 2 .

To see that the distance between vertices of $K_{h}^{(i j k)}$ and vertices of $K_{h}^{(r s t)}$ is at most 6 , we need to distinguish two cases:

Case 1. $h \leq \Delta\left(B_{\pi}\right)$.

Let $P=\left(x_{i j k}, S_{z_{\alpha}}\right)\left(u, S_{z_{\delta}}\right)\left(v, S_{z_{\theta}}\right)\left(x_{r s t}, S_{z_{\beta}}\right)$ be a 3-path between $x_{i j k}$ and $x_{r s t}$ in $G\left(B_{\pi}\right)$. Then, as vertices in $B_{\pi}$ from the same partite set are at distance at most 2 , there are $\Delta\left(B_{\pi}\right)$ 3-paths (each going through a different neighbor of $x_{i j k}$ in $S_{z_{\delta}}$ ) between $x_{i j k}$ and $x_{r s t}$; see Figure $14(a)$. Thus, adjacencies in $\hat{G}\left(K_{h}\right)$ can be organized in such a way that every vertex $y_{\gamma}^{(i j k)} \in K_{h}^{(i j k)}$ is linked to a copy $K_{h}^{(o p q)}$, where $K_{h}^{(o p q)}$ is at distance 3 from $K_{h}^{(r s t)}$; see Figure $14(b)$.

Thus, Condition 3a of Theorem 4.1 holds.

Case 2. $h=\Delta\left(B_{\pi}\right)+1$.

We can organize adjacencies in $\hat{G}\left(K_{h}\right)$ in such a way that there is exactly one vertex in each copy $K_{h}^{(i j k)}$ that is not connected to any copy $K_{h}^{(o p q)}$ at distance 3 from $K^{(r s t)_{h}}$. For each copy $K_{h}^{(i j k)}$, we take such a vertex as $y_{0}^{(i j k)}$, so there is a 6-path $P$ between $y_{0}^{(i j k)}$ and $y_{0}^{(r s t)}$ going through the subgraph $R$ of $H_{\Delta-1}$ (see Figure 2], $P=y_{0}^{(i j k)} x_{i j} x_{i} x x_{r} x_{r s} y_{0}^{(r s t)}$.

Furthermore, observe that Condition 3a holds for all vertices in $K_{h}^{(i j k)}$ except $y_{0}^{(i j k)}$.

We now present in Table 2 the features of each $H_{\Delta-1}\left(K_{h}\right)$ obtained by this approach, while in Tables 3, 4 and 5, we show the labeling of the vertices within a partite set in the graphs $C_{7}\left(I_{5}\right)$, 


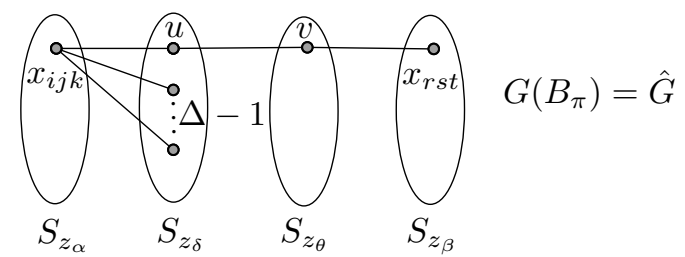

(a)

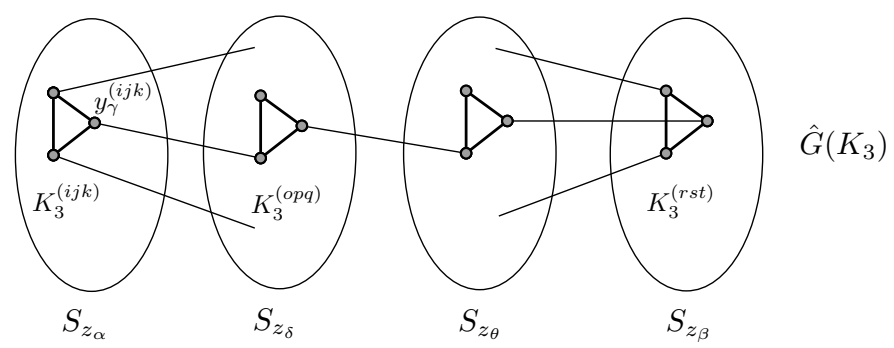

(b)

Figure 14: Path of length at most 6 between $y_{\gamma}^{(i j k)} \in K_{3}^{(i j k)}$ and any vertex in $K_{3}^{(r s t)}$. Note that $d\left(K_{3}^{(o p q)}, K_{3}^{(r s t)}\right)=3$.

$C_{5} \times F_{4}(B D)$ and $H\left(I_{8}\right)$, respectively.

Some of the graphs used to produce graphs $G\left(B_{\pi}\right)$ are the following:

$I_{\Delta-1}$ : Moore bipartite graph of diameter 3 and degree $\Delta$. Each such graph has a polarity [6].

$C_{5} \times F_{4}$ : Maximal graph of degree 3, diameter 3 and order 20, found by Green [18].

$B D$ : Bipartite graph of diameter 3, degree 7 and order 78 , found in [7]. This graph has a polarity.

$H$ : The unique graph of diameter 3, degree 3 and order 18, constructed by Faradžev [19]; see also [32].

Furthermore, note that

$H_{8}\left(K_{6}\right)$ : The cardinality of each block is either 27 or 28 . As the order of $\hat{G}=C_{7}\left(I_{5}\right)$ is $7 \times 31=$ 217 , by using Equation (3), $n\left(H_{8}\left(K_{6}\right)\right)=n\left(H_{8}\right)+217 \times 5=75893$.

$H_{11}\left(K_{8}\right)$ : The cardinality of each block is exactly 78 . As the order of $\hat{G}=C_{5} \times F_{4}(B D)$ is $20 \times 39=780$, by using Equation (3), $n\left(H_{11}\left(K_{8}\right)\right)=n\left(H_{11}\right)+780 \times 7=359772$. 
$H_{13}\left(K_{10}\right)$ : The cardinality of each block is either 109 or 110 . As the order of $\hat{G}=H\left(I_{8}\right)$ is $18 \times 73=1314$, by using Equation (3), $n\left(H_{13}\left(K_{10}\right)\right)=n\left(H_{13}\right)+1314 \times 9=816294$.

Table 2: Features of the graphs $H_{\Delta-1}\left(K_{h}\right)$ obtained by Approach 2.

\begin{tabular}{||c||c|c|c|c|c|c|c|c|c|c||}
\hline \hline$\Delta$ & $h$ & $M$ & $\delta$ & $M^{\prime}$ & $G$ & $B_{\pi}$ & Labeling of $G$ & Distribution of Labels in $\hat{G}$ & $H_{\Delta-1}\left(K_{h}\right)$ \\
\hline \hline 9 & 6 & 15 & 3 & 12 & $C_{7}$ & $I_{5}$ & See Fig. 15 & See Table 3 & & $H_{8}\left(K_{6}\right)$ \\
\hline 12 & 8 & 28 & 7 & 21 & $C_{5} \times F_{4}[18]$ & $B D[7]$ & See Fig. 15 & See Table 4 & $H_{11}\left(K_{8}\right)$ \\
\hline 14 & 10 & 36 & 9 & 27 & $H[19]$ & $I_{8}$ & See Fig. 15 & See Table 5 & $H_{13}\left(K_{10}\right)$ \\
\hline \hline
\end{tabular}
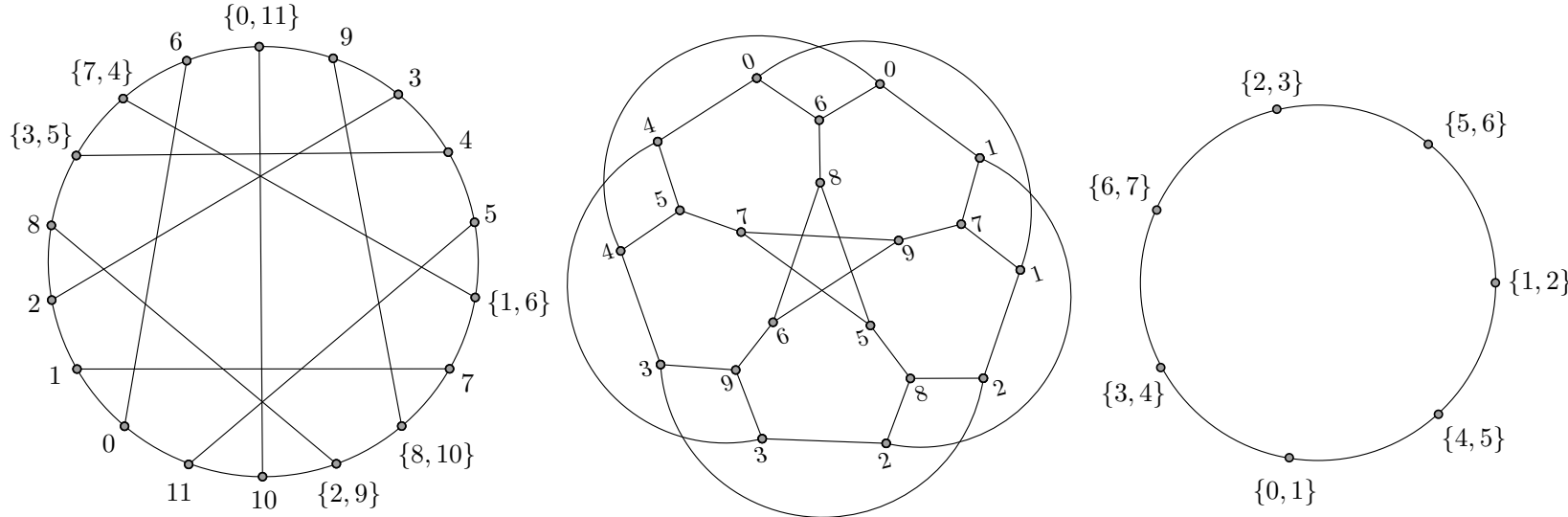

Figure 15: Labeling of the graphs $H, C_{5} \times F_{4}$ and $C_{7}$.

\section{Conclusion}

In this paper we have presented a new family of compound graphs of diameter 6 , denoted by $H_{\Delta-1}\left(K_{h}\right)$. At least five members of this family-namely, $H_{4}\left(K_{3}\right), H_{5}\left(K_{4}\right), H_{8}\left(K_{6}\right), H_{11}\left(K_{8}\right)$ and $H_{13}\left(K_{10}\right)$ - constitute graphs of largest known order of diameter 6 for their respective maximum degrees; see Table 6 . In order to obtain some of these new largest known graphs, we have defined a second new family of compound graphs, denoted by $G\left(B_{\pi}\right)$, and we proved that their maximum degree is $\Delta(G) \times \Delta\left(B_{\pi}\right)$, their order is $\frac{n(G) n\left(B_{\pi}\right)}{2}$, and their diameter is 
Table 3: Labeling of vertices within a partite set in $C_{7}\left(I_{5}\right)$.

\begin{tabular}{||c||c||}
\hline \hline Label of Vertex in $G=C_{7}$ & Distribution of Labels in $\hat{G}=C_{7}\left(I_{5}\right)$ \\
\hline \hline$\{0,1\}$ & 27 vertices take the label 0 and 4 vertices take the label 1 \\
\hline$\{1,2\}$ & 23 vertices take the label 1 and 8 vertices take the label 2 \\
\hline$\{2,3\}$ & 19 vertices take the label 2 and 12 vertices take the label 3 \\
\hline$\{3,4\}$ & 15 vertices take the label 3 and 16 vertices take the label 4 \\
\hline$\{4,5\}$ & 11 vertices take the label 4 and 20 vertices take the label 5 \\
\hline$\{5,6\}$ & 7 vertices take the label 5 and 24 vertices take the label 6 \\
\hline$\{6,7\}$ & 3 vertices take the label 6 and 28 vertices take the label 7 \\
\hline \hline
\end{tabular}

Table 4: Labeling of vertices within a partite set in $C_{5} \times F_{4}(B D)$.

\begin{tabular}{||c||c||}
\hline \hline Label of Vertex in $G=C_{5} \times F_{4}$ & Distribution of Labels in $\hat{G}=C_{5} \times F_{4}(B D)$ \\
\hline \hline$i$, for $i \in\{0,1,2,3,4,5,6,7,8,9\}$ & 39 vertices take the label $i$ \\
\hline
\end{tabular}

Table 5: Labeling of vertices within a partite set in $H\left(I_{8}\right)$.

\begin{tabular}{||c||c||}
\hline \hline Label of Vertex in $G=H$ & Distribution of Labels in $\hat{G}=H\left(I_{8}\right)$ \\
\hline \hline$i, 0 \leq i \leq 11$ & 73 vertices take the label $i$ \\
\hline$\{a, b\}$, for $0 \leq a, b \leq 11$ and $a<b$ & $\begin{array}{c}37 \text { vertices take the label } a \\
\text { and } 36 \text { vertices take the label } b\end{array}$ \\
\hline \hline
\end{tabular}

Table 6: Orders of the new largest known graphs $H_{\Delta-1}\left(K_{h}\right)$, for $\Delta \leq 14$.

\begin{tabular}{||c||c||c|c||}
\hline$\Delta$ & Previous Largest Known Order & New Largest Known Order & Graph \\
\hline \hline 5 & 2766 & $\mathbf{2 7 7 2}$ & $H_{4}\left(K_{3}\right)$ \\
6 & 7908 & $\mathbf{7 9 1 7}$ & $H_{5}\left(K_{4}\right)$ \\
\hline 9 & 75828 & $\mathbf{7 5 8 9 3}$ & $H_{8}\left(K_{6}\right)$ \\
\hline 12 & 359646 & $\mathbf{3 5 9 7 7 2}$ & $H_{11}\left(K_{8}\right)$ \\
\hline 14 & 816186 & $\mathbf{8 1 6 2 9 4}$ & $H_{13}\left(K_{10}\right)$ \\
\hline \hline
\end{tabular}


$\max \left\{D(G), D\left(B_{\pi}\right)\right\}$

When analyzing Table 6, note that the previously largest known graphs were obtained in [27] and in [23], and we have kept the same terminology as in those papers.

\section{References}

[1] E. Bannai and T. Ito, On finite Moore graphs, J Fac Sci Tokyo Univ 20 (1973), 191-208.

[2] J. C. Bermond, C. Delorme, and G. Fahri, Large graphs with given degree and diameter III, Proc Coll Cambridge 1981, Ann Discrete Math 13 (1982), 23-32.

[3] J. C. Bermond, C. Delorme, and G. Fahri, Large graphs with given degree and diameter II, J Comb Theory B 36 (1984), 32-48.

[4] J. C. Bermond, C. Delorme, and J. J. Quisquater, Grands graphes non dirigés de degré et diamètre fixés, Ann Discrete Math 17 (1982), 65-73.

[5] J. C. Bermond, C. Delorme, and J.J. Quisquater, Strategies for interconnection networks: Some methods from graph theory, J Parallel and Distrib Comp 3 (1986), 433-449.

[6] N.I. Biggs, Algebraic graph theory, Second edition, Cambridge University Press, Cambridge, 1993.

[7] J. Bond and C. Delorme, New large bipartite graphs with given degree and diameter, Proc 11th British Combinatorial Conference, London, 1987, Ars Combin 25-C (1988), 123-132.

[8] D. Buset, Maximal cubic graphs with diameter 4, Discrete Appl Math 101 (2000), 53-61.

[9] E. Canale and J. Gómez, Asymptotically large $(\Delta, D)$-graphs, Discrete Appl Math 152 (2005), 89-108.

[10] F. Comellas and J. Gómez, New large graphs with given degree and diameter, Graph theory, Combinatorics and Applications: Proc 7th Quadrennial International Conference on the Theory and Applications of Graphs Vol. 1, 1995, pp. 221-233. 
[11] C. von Conta, Torus and other networks as communication networks with up to some hundred points, IEEE Trans Comput C 32 (1983), 657-666.

[12] R. M. Damerell, On Moore graphs, Proc Cambridge Phil Soc 74 (1973), 227-236.

[13] C. Delorme, Grands graphes de degré et diamètre donnés, Europ J Combinatorics 6 (1985), 291-302.

[14] C. Delorme, Examples of products giving large graphs with given degree and diameter, Discrete Appl Math 37/38 (1992), 157-167.

[15] C. Delorme and J. Gómez, Some new large compound graphs, Europ J Combinatorics 23 (2002), 539-547.

[16] R. Diestel, Graph theory, Third edition, Springer-Verlag, New York, 2005.

[17] K.W. Doty, Large regular interconnection networks, Proc 3rd International Conference on Distributed Computing Systems, Miami, IEEE Press, 1982, pp. 312-317.

[18] B. Elspas, Topological constraints on interconnection-limited logic, Proc IEEE 5th Symposium on Switching Circuit Theory and Logical Design, IEEE Vol. S-164, 1964, pp. 133-147.

[19] I. A. Faradžev, Constructive enumeration of combinatorial objects, Problemes Combinatoires et Theorie des Graphes Colloque Internat, CNRS 260, CNRS Paris, 1978, pp. 131-135.

[20] M. A. Fiol and J. Fàbrega, Algunos grafos compuestos, Stochastica VII 2 (1983), 137-143.

[21] C. D. Godsil and G. F. Royle, Algebraic graph theory, Springer-Verlag, New York, 2001.

[22] J. Gómez, Generalized compound graphs, Ars Combin 29-B (1990), 33-53.

[23] J. Gómez, On large $(\Delta, 6)$-graphs, Networks 46 (2005), 82-87.

[24] J. Gómez and M. A. Fiol, Dense compound graphs, Ars Combin 20-A (1985), 211-237.

[25] J. Gómez, M. A. Fiol, and O. Serra, On large $(\Delta, D)$-graphs, Discrete Math 114 (1993), 219-235. 
[26] J. Gómez and M. Miller, Two new families of large compound graphs, Networks 47 (2006), 140-146.

[27] J. Gómez, I. Pelayo, and C. Balbuena, New large graphs with given degree and diameter six, Networks 34 (1999), 154-161.

[28] A. J. Hoffman and R. R. Singleton, On Moore graphs with diameter 2 and 3, IBM J Res Develop 4 (1960), 497-504.

[29] L. K. Jørgensen, Diameters of cubic graphs, Discrete Appl Math 37/38 (1992), 347-351.

[30] M. Miller and J. Širán̆, Moore graphs and beyond: A survey of the degree/diameter problem, The Electronic Journal of Combinatorics DS14 (2005), 1-61.

[31] S. G. Molodtsov, "Largest graphs of diameter 2 and maximum degree 6", General Theory of Information Transfer and Combinatorics, R. Ahlswede (Editor), Lecture Notes in Computer Science, Vol. 4123, Springer Berlin/Heidelberg, 2006, pp. 853-857.

[32] G. Pineda-Villavicencio and M. Miller, On graphs of maximum degree 3 and defect 4, Journal of Combinatorial Mathematics and Combinatorial Computing, to appear.

[33] J. J. Quisquater, Structures d'interconnexion: Constructions et applications, Thèse d'etat, LRI, Université de Paris Sud, Orsay, 1987.

[34] Table of the largest known graphs for maximum degrees $3 \leq \Delta \leq 16$ and diameters $2 \leq$ $D \leq 10$, webpage. http://maite71.upc.es/grup_de_grafs/grafs/taula_delta_d.html.

[35] J. Xu, Topological structure and analysis of interconnection networks, Kluwer Academic Publishers, Dordrecht, 2001. 